PHYSICAL REVIEW D 92, 069901(E) (2015)

\title{
Erratum: Nonexistence of black holes with noncanonical scalar fields [Phys. Rev. D 89, 084056 (2014)]
}

\author{
Alexander A. H. Graham and Rahul Jha \\ (Received 26 August 2015; published 9 September 2015)
}

DOI: 10.1103/PhysRevD.92.069901

PACS numbers: 04.70.Bw, 04.50.Kd, 99.10.Cd

The following corrections apply to our paper:

(1) In Eqs. (2), (8), (10), (12), and (17), the integrals should be four-dimensional integrals, running from the black hole horizon to infinity.

(2) In Secs. II-V, when referring to our theorem, "stationary" should be replaced by "static or stationary and axisymmetric."

(3) In Sec. IV B, the correct conditions where the theorem applies are the following: $\lambda>0$ and $\phi \geq 0$ or $\lambda<0$ and $\phi \leq 0$. 